\title{
EFEITO DA ADIÇÃO DE GENGIBRE (Zingiber officinale) EM PÓ SOBRE O DESEMPENHO DE FRANGOS DE CORTE NA FASE INICIAL
}

Vilson Matias Pinto1; Paulo Ricardo de Sá da Costa Leite²; Mônica Maria de Almeida Brainer; Brena Cristine Rosário Silva ${ }^{4}$; Leidyanne Guimarães Silva ${ }^{4}$.

${ }^{1}$ Estudante de Zootecnia, Instituto Federal Goiano - Campus Ceres; ${ }^{2}$ Professor do Curso de Zootecnia, Instituto Federal Goiano - Campus Ceres; ${ }^{3}$ Professora de Curso de Zootecnia, Instituto Federal Goiano - Campus Ceres; ${ }^{4}$ Estudante de Zootecnia, Instituto Federal Goiano - Campus Ceres.

\section{RESUMO}

Objetivou-se avaliar o desempenho de frangos alimentados com adição de gengibre (Zingiber officinale) em pó na fase inicial de criação. Foram utilizados 630 pintinhos de corte distribuídos em delineamento inteiramente casualizado, com cinco tratamentos e sete repetições com dezoito aves por unidade experimental. Os tratamentos consistiram de dieta referência com milho e farelo de soja e adição de diferentes níveis de gengibre em pó, sendo cinco tratamentos, composto por controle positivo, controle negativo e três níveis de gengibre $(0,50 \% ; 0,75 \% ; 1,0 \%)$. Foram avaliados, no final de cada fase de criação, o consumo de ração, ganho de peso, conversão alimentar, peso final, comprimento e peso do intestino. Os dados obtidos foram submetidos à análise de variância e as médias à análise de regressão com $5 \%$ de significância. Não foram observados efeitos dos tratamentos sobre as variáveis de desempenho na fase inicial de criação. Com este resultado, aos vinte e um dias de idade, foi possível observar o potencial de substituição dos melhoradores de desempenho pelo gengibre em pó. A inclusão de até $1 \%$ de gengibre em pó não afetou o desempenho das aves proporcionando resultados semelhantes ao melhorador de desempenho.

Palavras-chave: aditivos; antimicrobianos; melhorador de desempenho.

\section{EFFECT OF THE ADDITION OF GINGER (Zingiber officinale) POWDER ONTHE PERFORMANCE OF EARLY-STAGE CHICKEN}

\begin{abstract}
The objective of this study was to evaluate the production performance of broilers with the addition of ginger (Zingiber officinale) in broiler diets in starter phases. Were used 630 broiler chicks in a completely randomized design with five treatments and seven repetitions with eighteen birds each. The treatments consist of a reference diet with corn and soybean meal and adding different levels of powdered ginger, five treatments, consisting of positive control, negative control and three levels of ginger $(0.50 \%, 0.75 \% ; 1.0 \%)$. Were evaluated the end of each phase, feed intake, weight gain, feed conversion, body weight, pancreatic weight and length and weight of the intestine. The data will be submitted to analysis of variance and means to regression analysis with $5 \%$ significance. No effects of the treatments on the performance variables were observed in the initial stage of creation. With this result, at twenty-one days old, we observed the potential for substitution of growth promoters for powdered ginger. The inclusion of up to $1 \%$ of powdered ginger did not affect bird performance providing results similar to positive control treatments with growth promoter.
\end{abstract}

Keywords: additives; antimicrobials; performance enhancement. 


\section{INTRODUÇÃO}

A utilização de melhoradores de desempenho à base de agentes antibacterianos na dieta é uma prática frequente e rotineira na avicultura por propiciar maior desempenho produtivo. De acordo com Brumano e Gattás (2009), os antimicrobianos têm como finalidade prevenir, reduzir ou controlar agentes prejudiciais ao processo digestivo, melhorando o desempenho, sendo classificados como pró-nutrientes e pertencentes ao grupo de melhoradores de desempenho.

No entanto, o uso desses antibióticos vem sofrendo restrições nos últimos anos, devido à possibilidade de seleção de microrganismos resistentes, desenvolvimento de resistência bacteriana cruzada em humanos e devido à exigência de produtos livres de resíduos de antibióticos pelo mercado consumidor (TRAESEL et al., 2011; PASQUALI, 2014).

A União Européia, preocupada com esta questão, decidiu banir o uso de qualquer tipo de antibiótico melhorador de desempenho na nutrição animal. Já no Brasil, o Ministério da Agricultura, Pecuária e Abastecimento (MAPA), vem regulamentando os antimicrobianos que são permitidos na alimentação animal no Brasil, gerando a proibição de alguns, como: avoparcina e tetraciclinas (1998), cloranfenicol (2003), carbadox (2005), espiramicina e eritromicina (2012), sulfato de colistina (2016).

Nesse sentido, é importante o estudo de novas tecnologias e pesquisas que possibilitem alternativas para a substituição dos antibióticos, destacando-se os aditivos fitogênicos. Esses aditivos, como os extratos herbais e vegetais, são algumas alternativas, fazendo parte de uma classe de produtos que poderá substituir os agentes antimicrobianos (FUKAYAMA, 2005). Dentre os aditivos fitogênicos, destaca-se o gengibre (Zingiber officinale) cujo rizoma é amplamente comercializado em função de seu emprego alimentar e industrial, além de ser utilizada na medicina popular, em função de melhorar a secreção gástrica, sua atividade anti-inflamatória e antibacteriana (ELPO e NEGRELLE, 2004; YAMAUCHI et al.,2009).

A inclusão do gengibre em pó na alimentação de frangos de corte pode melhorar a conversão alimentar, além de trazer diversos benefícios em termos de estado antioxidante e parâmetros sanguíneos como níveis de hemoglobina e células vermelhas, bem como pode melhorar o sistema imunológico e a microbiota intestinal das aves (TEKELI et al.,2006; SELIM et al., 2013).

Estudos de Herawati (2010) e Mohammed e Yusuf (2011) relataram efeitos do gengibre na ração de aves, com melhora na digestibilidade dos nutrientes da ração e no desempenho. No trabalho de Elmakki et al. (2013), que avaliaram o efeito da inclusão de gengibre em rações para frangos de corte da linhagem Cobb e concluíram que os frangos conseguem tolerar até $0,75 \%$ de gengibre na ração sem causar efeitos contrários no desempenho das aves.

Diante deste contexto, objetivou-se estudar a adição de gengibre (Zingiber officinale) em pó em rações de frangos de corte na fase inicial de criação.

\section{MATERIAIS E MÉTODOS}

O projeto foi realizado no Setor de Avicultura do Instituto Federal Goiano - Campus Ceres, Ceres/GO e foi aprovado pela Comissão de ética no uso de animais (CEUA) com número de protocolo 020/2015.

Foram utilizados 630 pintinhos de corte machos da linhagem Cobb, distribuídos em delineamento inteiramente casualizado com cinco tratamentos, sete repetições e 18 aves por unidade experimental.

As aves foram alojadas no aviário experimental do Setor de Avicultura do IF Goiano. As instalações consistiram em um galpão de alvenaria telado com cortinas, dividido em 35 boxes de $4 \mathrm{~m}^{2}$ com divisórias de madeira e tela, com um bebedouro pendular e comedouro tubular. Os boxes foram cobertos com cama de frango reutilizada de lotes de aves caipiras, como forma de proporcionar um desafio sanitário às aves. 
As rações foram elaboradas com milho e farelo de soja seguindo as recomendações de Rostagno et al. (2011), para cada fase de criação: pré-inicial (1 a 7 dias) e inicial (8 a 21 dias) (Tabela 1). $O$ gengibre em pó foi adquirido em comércio especializado e adicionado na ração basal em substituição ao inerte de forma a atender os percentuais de $0,50 \%, 0,75 \%$ e 1,0\% de inclusão, níveis baseados nos estudos de Herawati (2010). Os tratamentos empregados são descritos a seguir:

Tratamento 1 - ração basal (RB) com melhorador de desempenho (bacitracina de zinco) e sem gengibre em pó (controle positivo);

Tratamento 2 - ração basal (RB) sem melhorador de desempenho e sem gengibre em pó (controle negativo);

Tratamento 3 - RB sem melhorador de desempenho e com 0,50\% de gengibre em pó;

Tratamento 4 - RB sem melhorador de desempenho com $0,75 \%$ de gengibre em pó;

Tratamento 5 - RB sem melhorador de desempenho com 1,0\% de gengibre em pó.

Tabela 1. Composição e valor nutricional das dietas experimentais

\begin{tabular}{|c|c|c|c|c|}
\hline \multirow[t]{2}{*}{ Ingredientes (g/kg) } & \multirow{2}{*}{$\begin{array}{c}\text { RB (Positiva) } \\
\text { Pré-inicial }\end{array}$} & \multicolumn{2}{|c|}{ RB (Negativa) } & \multirow[b]{2}{*}{ Inicial } \\
\hline & & Inicial & Pré-inicial & \\
\hline Milho & 533,07 & 576,21 & 533,44 & 576,21 \\
\hline Soja Farelo $45 \%$ & 387,52 & 350,00 & 350,00 & 350,00 \\
\hline Óleo de Soja & 27,920 & 27,03 & 27,03 & 27,03 \\
\hline Fosfato Bicálcico & 19,120 & 15,04 & 15,04 & 15,04 \\
\hline Inerte & 10,00 & 10,00 & 10,00 & 10,00 \\
\hline Calcário & 7,96 & 8,24 & 8,24 & 8,24 \\
\hline Sal Comum & 5,07 & 4,82 & 4,82 & 4,82 \\
\hline DL- Metionina & 3,580 & 2,89 & 2,89 & 2,89 \\
\hline L- Lisina HCL (79\%) & 2,74 & 2,72 & 2,72 & 2,72 \\
\hline Premix vitamínico aves ${ }^{1}$ & 1,00 & 1,00 & 1,00 & 1,00 \\
\hline Cloreto de colina $70 \%$ & 0,60 & 0,60 & 0,60 & 0,60 \\
\hline Coxistac & 0,55 & 0,55 & 0,55 & 0,55 \\
\hline Premix mineral aves ${ }^{2}$ & 0,50 & 0,50 & 0,50 & 0,50 \\
\hline Bacitracina de zinco & 0,37 & 0,37 & 0,00 & 0,00 \\
\hline Total & 1000,00 & 1000,00 & 1000,00 & 1000,00 \\
\hline \multicolumn{5}{|l|}{ Composição Nutricional (g/kg) } \\
\hline Proteína bruta & 222,00 & 208,00 & 222,00 & 208,00 \\
\hline Energia (mcal/Kg) metabolizável & 29,50 & 30,00 & 29,50 & 30,00 \\
\hline Lisina digestível aves & 13,10 & 12,20 & 13,10 & 12,20 \\
\hline Cálcio & 9,20 & 8,19 & 9,20 & 8,19 \\
\hline Fósforo Disponível & 4,70 & 3,91 & 4,70 & 3,91 \\
\hline Metionina + cistina digestível aves & 9,44 & 8,46 & 9,44 & 8,46 \\
\hline Metionina digestível aves & 6,47 & 5,62 & 6,45 & 5,62 \\
\hline Potássio & 8,64 & 8,08 & 8,64 & 8,08 \\
\hline Sódio & 2,20 & 2,10 & 2,20 & 2,10 \\
\hline Treonina digestível aves & 0,00 & 7,05 & 0,00 & 7,05 \\
\hline
\end{tabular}

${ }^{1}$ Suplemento vitamínico contendo: Ácido fólico (min) 700 mg, Ácido Pantatênico (min) 8000 mg, Biotina (min) 60 mg, Niacina (min) 30 g, Selênio (min) 400 mg, Vit. A (min) 8.000.000 UI, Vit. B1 (min) 3000 mg, Vit. B12 (min) 10.000 cg, Vit. B2 (min) 4000 mg, Vit. B6 (min) 2000 mg, Vit. D3 (min) 2.000.000 UI, Vit. E (min) 15.000 UI, Vit. K3 (min) 2000 mg. ${ }^{2}$ Suplemento mineral contendo: Zinco (min) 125 g. Cobre (min) 12,6 g. lodo (min) 2.520 mg. Ferro (min) 105 g. Manganês (min) $126 \mathrm{~g} .{ }^{3}$ Anticoccidiano: Salinomicina sódica micelial (min) $12 \mathrm{~g}$. 
O aquecimento das aves foi feito através de um sistema elétrico, sendo colocada uma lâmpada incandescente de $60 \mathrm{~W}$ por unidade experimental e com auxílio de campânulas até os 14 dias. A temperatura e umidade relativa foram registradas durante todo o período experimental com auxílio de um termo higrômetro. As aves receberam água e ração à vontade.

Ao final de cada fase experimental ( 1 a 7 dias, 8 a 21 dias), as aves e as sobras de ração foram pesadas para avaliação do peso médio, ganho de peso, consumo de ração e a conversão alimentar. Para a verificação do desempenho, o ganho de peso foi obtido através da diferença entre o peso inicial e o peso final de cada período. O consumo de ração foi calculado considerando-se a ração fornecida e as sobras de rações nos comedouros, assim como a conversão alimentar foi obtida por meio da divisão do consumo de ração e do peso das aves a cada intervalo.

A mortalidade foi verificada diariamente em função da quantidade de aves em cada parcela experimental. Para a biometria dos órgãos do sistema digestório cinco aves por tratamento foram escolhidas aleatoriamente e eutanasiadas por deslocamento cervical para a determinação do peso relativo do fígado, pâncreas e intestino, além da mensuração do comprimento do intestino.

Os dados obtidos foram submetidos à análise de variância e quando significativo às médias à análise de regressão com 5\% de significância utilizando o programa ASSISTAT (2000).

\section{RESULTADOS E DISCUSSÃO}

A média de temperatura máxima e mínima, observada foi de $34,77 \circ \mathrm{C}$ e $24,35 \circ \mathrm{C}$, respectivamente, exibindo umidade máxima $(41,2 \%)$ e mínima $(22,7 \%)$. A adição dos diferentes níveis de gengibre em pó proporcionou resultados semelhantes ao tratamento com melhorador de desempenho (Tabela 2). Com estes resultados, foi possível observar o potencial de substituição dos melhoradores de desempenho pelo gengibre em pó na ração de frangos de corte na fase préinicial.

A utilização do gengibre em pó na dieta não apresentou efeito no consumo de ração na primeira semana resultado semelhante ao de Elmakki et al. (2013). No trabalho de Shiva et al. (2012), a adição do óleo essencial de orégano (Origanum vulgare) e gengibre seco (Zingiber officinale) na fase inicial de criação de frangos macho da linhagem Coob, não proporcionou efeito dos aditivos fitogênicos sobre o peso corporal, consumo de ração e conversão alimentar.

Tabela 2. Desempenho de frangos de corte submetidos a diferentes níveis de inclusão de gengibre (Zingiber officinale) na ração no período de 1 a 7 dias de vida

\begin{tabular}{llllllc}
\hline \multirow{2}{*}{ Variáveis } & \multicolumn{7}{c}{ Níveis de adição de gengibre } & \multirow{2}{*}{ CV (\%) } \\
\cline { 2 - 6 } & $\begin{array}{l}\text { Controle } \\
\text { Positivo }\end{array}$ & $\begin{array}{l}\text { Controle } \\
\text { Negativo }\end{array}$ & $0,50 \%$ & $0,75 \%$ & $1 \%$ & \\
\hline $\mathrm{CR}^{1}$ & 0,192 & 0,196 & 0,187 & 0,187 & 0,187 & 8,00 \\
$\mathrm{GP}^{2}$ & 0,147 & 0,146 & 0,142 & 0,143 & 0,142 & 9,93 \\
$\mathrm{CA}^{3}$ & 1,054 & 1,070 & 1,050 & 1,057 & 1,047 & 13,31 \\
$\mathrm{PF}^{4}$ & 0,184 & 0,183 & 0,179 & 0,180 & 0,179 & 7,98 \\
\hline
\end{tabular}

${ }^{1} \mathrm{CR}$ - Consumo de Ração; ${ }^{2} \mathrm{GP}$ - Ganho de Peso; ${ }^{3} \mathrm{C} 4$ - Conversão Alimentar; CV - Coeficiente de Variação; ${ }^{4} \mathrm{PF}$ Peso Final. CV - Coeficiente de Variação.

Com relação aos dados da fase inicial (8 a 21 dias) não foram observadas efeito sobre o peso final, conversão alimentar, ganho de peso e consumo de ração das aves entre os tratamentos (Tabela 3). 
Tabela 3. Desempenho de frangos de corte submetidos a diferentes níveis de inclusão de gengibre (Zingiber officinale) na ração no período de 8 a 21 dias de vida

\begin{tabular}{lllllll}
\hline \multirow{2}{*}{ Variáveis } & \multicolumn{7}{c}{ Níveis de adição de gengibre } & \multirow{2}{*}{ CV (\%) } \\
\cline { 2 - 6 } & $\begin{array}{l}\text { Controle } \\
\text { Positivo }\end{array}$ & $\begin{array}{l}\text { Controle } \\
\text { Negativo }\end{array}$ & $0,50 \%$ & $0,75 \%$ & $1 \%$ & \\
\hline $\mathrm{CR}^{1}$ & 1,456 & 1,404 & 1,438 & 1,431 & 1,426 & 6,97 \\
$\mathrm{GP}^{2}$ & 0,849 & 0,843 & 0,851 & 0,842 & 0,851 & 6,10 \\
$\mathrm{CA}^{3}$ & 1,417 & 1,374 & 1,398 & 1,406 & 1,384 & 9,17 \\
$\mathrm{PF}^{4}$ & 1,033 & 1,026 & 1,030 & 1,022 & 1,030 & 6,01 \\
\hline
\end{tabular}

${ }^{1} \mathrm{CR}$ - Consumo de Ração; ${ }^{2} \mathrm{GP}$ - Ganho de Peso; ${ }^{3} \mathrm{C} 4$ - Conversão Alimentar; CV - Coeficiente de Variação; ${ }^{4} \mathrm{PF}$ Peso Final. CV - Coeficiente de Variação.

Da mesma forma, na pesquisa de Koiyama et al. (2014), com mistura de aditivos fitogênicos, dentre eles o gengibre, foi observado que não houve diferença sobre o ganho de peso, conversão alimentar e consumo de ração de frangos na fase inicial de criação. Resultado semelhante foi observado por MOHAMMED e YUSUF (2011) quando estudaram a inclusão de diferentes níveis $(0,250,500$ e $750 \mathrm{~g} / 100 \mathrm{~kg})$ de gengibre em rações de frango e também não observaram efeito dos tratamentos sobre os resultados de consumo de ração, ganho de peso, conversão alimentar e peso final. Em outro estudo Elmakki et al. (2013) relataram que frangos da linhagem Cobb conseguem tolerar até $0,75 \%$ de inclusão de gengibre em rações sem causar efeitos contrários no ganho de peso, conversão alimentar e peso final.

Entretanto, na pesquisa de HERAWATI (2010) foi observado que frangos da linhagem Hubbard quando alimentados com rações suplementadas com $2 \%$ de gengibre obtiveram maior peso corporal. Diferente deste estudo, o maior percentual utilizado nesta pesquisa foi de $1 \%$, 0 que não foi suficiente para proporcionar melhor resultado de desempenho, sugerindo que aves da linhagem Cobb também podem tolerar maiores níveis.

Os diferentes resultados encontrados nas pesquisas podem está relacionados ao percentual de inclusão de cultivar do gengibre utilizado (MOHAMMED e YUSUF, 2011). Zhang et al. (2009), observaram que as aves suplementadas com gengibre não apresentaram rendimento de carcaça, taxa de conversão alimentar e ganho de peso final, em comparação com o tratamento controle.

Outro fator que pode influenciar os resultados com os aditivos fitogênicos é o desafio sanitário a que as aves são submetidas. Neste estudo foi proposto como desafio sanitário a reutilização de cama de aves caipiras, pois segundo alguns autores (SHILA et al., 2012; KOIYAMA et al., 2014; BRUGALLI, 2003) para o efeito dos aditivos fitogênicos é importante ter algum desafio sanitário. De acordo com Leite et al. (2012) frangos submetidos a desafio sanitário podem responder melhor aos antimicrobianos, se comparados aqueles alojados em baterias, com melhores condições sanitárias e ambientais. Porém os resultados obtidos demonstraram que o desafio não foi suficiente, para um efeito positivo do gengibre como substituto dos melhoradores de desempenho.

Em relação aos pesos relativos de vísceras e comprimento de intestino delgado, as aves que foram abatidas com vinte e um dias de idade não apresentaram efeito dos tratamentos, com exceção ao comprimento do intestino grosso, em que os níveis de 0,50\% e 0,75\% de adição de gengibre proporcionaram resultados semelhantes ao controle positivo (Tabela 4). 
Tabela 4. Médias de pesos relativos de vísceras e comprimento de intestinos de frangos de corte submetidos a diferentes níveis de inclusão de gengibre na dieta aos 21 dias de vida

\begin{tabular}{lllllll}
\hline \multirow{2}{*}{ Variáveis } & \multicolumn{5}{c}{ Níveis de adição de gengibre } \\
\cline { 2 - 6 } & $\begin{array}{l}\text { Controle } \\
\text { Positivo }\end{array}$ & $\begin{array}{l}\text { Controle } \\
\text { Negativo }\end{array}$ & $0,50 \%$ & $0,75 \%$ & $1 \%$ & CV (\%) \\
\hline $\mathrm{CID}^{1}$ & 1,442 & 1,372 & 1,622 & 1,210 & 1,496 & 19,87 \\
$\mathrm{CIG}^{2}$ & $0,348^{\mathrm{a}}$ & $0,238^{\mathrm{c}}$ & $0,298^{\mathrm{ab}}$ & $0,298^{\mathrm{ab}}$ & $0,262^{\mathrm{bc}}$ & 9,98 \\
$\mathrm{PP}^{3}$ & 0,004 & 0,004 & 0,004 & 0,003 & 0,004 & 18,61 \\
$\mathrm{PF}^{4}$ & 0,032 & 0,030 & 0,028 & 0,030 & 0,028 & 14,87 \\
\hline${ }^{1} \mathrm{CID}$ - Comprimento do Intestino Delgado; ClG - Comprimento do Intestino Grosso; PP - Peso do Pâncreas; PF - \\
\multicolumn{7}{l}{ Peso do Fígado. CV - Coeficiente de Variação. }
\end{tabular}

O gengibre (Zingiber officinale) destaca-se entre os fitogênicos utilizados na alimentação animal, pois é considerado antibacteriano, além de proporcionar o desenvolvimento de bactérias benéficas na microbiota intestinal. Segundo Tekeli et al. (2006) a utilização de gengibre em rações de frangos proporciona maior número de unidades formadoras de colônias de Lactobacillus, na qual são consideradas bactérias benéficas, oferecendo assim melhor saúde intestinal. Assim com também Incharoen e Yamauchi (2009), que mostraram um grande número de bactérias desejáveis, no epitélio de aves recebendo uma dieta com gengibre, além de apresentarem maiores microvilosidades intestinais e área de superfície absortiva.

Com estes resultados pode-se observar que, até o vigésimo primeiro dia de idade, a inclusão de até $1 \%$ de gengibre em pó não afetou o desempenho das aves, proporcionando resultados semelhantes ao tratamento controle positivos, com melhorador de desempenho. Portanto, embora o gengibre não tenha tido efeito significativo, ele proporcionou os mesmos resultados dos antimicrobianos.

\section{CONCLUSÃO}

A inclusão de até $1 \%$ de gengibre em pó em ração inicial de frangos de corte, não aparentou nenhum efeito tóxico, ou seja, não houve nenhum efeito prejudicial para o desempenho das aves.

\section{AGRADECIMENTOS}

Ao Instituto Federal Goiano Campus Ceres pela bolsa PIBIC de Iniciação Científica

\section{COMITÊ DE ÉTICA E BIOSSEGURANÇA}

A Comissão de Ética no Uso de Animais - CEUA IF Goiano, na sua reunião de 06/11/2015, aprovou os procedimentos éticos apresentados no Protocolo no $020 / 2015$, do projeto intitulado: "Gengibre (Zingiber officinale) em ração de frangos de corte".

\section{REFERÊNCIAS}

BRASIL. Ministério da Agricultura, Pecuária e Abastecimento. Instrução Normativa 45, de 22 de novembro de 2016. Câmara de regulação do mercado de medicamentos. Diário Oficial [da] Republica Federativa do Brasil. Brasília, DF, 2016.

BRUGALLI, I. Alimentação alternativa: a utilização de fitoterápicos ou nutracêuticos como moduladores da imunidade e desempenho animal. In: SIMPÓSIO SOBRE MANEJO E NUTRIÇÃO DE AVES E SUÍNOS. Anais... Campinas: CBNA, 2003. p.167-182. 
BRUMANO, G.; GATTÁS, G. Implicações sobre o uso de antimicrobianos em rações de monogástricos. Revista Eletrônica Nutritime, v.6, n.3, 2009, p. 953-959.

ELMAKKI, A. M.; ABDELATTI, K. A.; DOUSA, B. M.; ELAGIB, H. A. A.; MALIK, H. E. E.; ELAMIN, K. M. Effects of dietary ground ginger (Zingiber Officinale) root additive on broiler performance. Global Journal of Animal Scientific Research. Vol 1, N 1, 2013.

ELPO, E. R. S.; NEGRELLE, R. R. B. Zingiber officinale Roscoe: aspectos botânicos e ecológicos. Visão Acadêmica, Curitiba, v. 5, n. 1, p. 27-32, Jan.- Jun./2004. DOI: https://doi.org/10.5380/acd.v5i1.539

FUKAYAMA, E. H.; BERTECHINI, A. G.; GERALDO, A.; KATO, R. K.; MURGASET, L. D. S. Extrato de Orégano como Aditivo em Rações para Frangos de Corte. Revista Brasileira de Zootecnia., v.34, n.6, p.2316-2326, 2005. https://doi.org/10.1590/S1516-35982005000700018

HERAWATI, M. The Effect of Red Ginger as Phytobiotic on Body Weight Gain, Feed Conversion and Internal Organs Condition of Broiler. International Journal of Poultry Science, v.9, p.963 967.2010. https://doi.org/10.3923/ijps.2010.963.967.

INCHAROEN, T; YAMAUCHI, K. Production performance, egg quality and intestinal histology in laying hens fed dietary dried fermented ginger. International Journal of Poultry Science, v.8, p.1078-1085, 2009. https://doi.org/10.3923/ijps.2009.1078.1085.

KOIYAMA; N. T. G.; ROSA, A. P.; PADILHA, M. T. S.; BOEMO, L. S.; SCHER, A.; MELO, A. M. S.; FERNANDES, M. O. Desempenho e rendimento de carcaça de frangos de corte alimentados com mistura de aditivos fitogênicos na dieta. Pesquisa agropecuária brasileira, Brasília, v.49, n.3, p.225-231, mar. 2014. https://doi.org/10.1590/S0100-204X2014000300009.

LEITE, P. R. S. C.; MENDES, F. R.; PEREIRA, M. L. R.; LIMA, H. J. D.; LACERDA, M. J. R. Aditivos fitogênicos em rações de frangos. Enciclopédia Biosfera. Goiânia: UFG, 2012.

MOHAMMED, A. A.; YUSUF, M.; Evaluation of ginger (Zingiber officinale) as a feed additive in broiler diets. Livestock Research for Rural Development, 2011.

PASQUALI, G. A. M.; PIMENTA, G. E. M. Aditivos fitogênicos: uma alternativa ao uso de antibióticos melhoradores de desempenho na alimentação de aves. Enciclopédia Biosfera, Centro Científico Conhecer - Goiânia, v.10, n.18; p. 2014.

ROSTAGNO, H. S.; ALBINO, L. F. T.; DONZELLE, J. F. GOMES, P. C.; FERREIRA, A. S.; OLIVEIRA, R. F. D.; LOPES, D. C., BARRETO, S. L. T. EUCLIDES, R. F. Tabelas brasileiras para aves e suínos. Composição de alimentos e exigências nutricionais. 3. ed. Viçosa: UFV, 2011. 252 p.

SELIM, N. A.; YOUSSEF, S. F.; ABDEL-SALAM, A. F.; NADA, S. A. Evaluation of Some Natural Antioxidant Sources in Broiler Diets: 1-Effect on Growth, Physiological, Microbiological and Immunological Performance of Broiler Chicks. International Journal of Poultry Science 12 (10): 561-571, 2013. https://doi.org/10.3923/ijps.2013.561.571.

SHIVA, C.; SAMUEL BERNAL, S.; SAUVAIN, M.; CALDAS, J.; KALINOWSKI, J.;FALCÓN, N.; ROJAS, R. Evaluación del aceite esencial de orégano (Origanum vulgare) y extracto deshidratado de jengibre 
(Zingiber officinale) como potenciales promotores de crecimiento en pollos de engorde. Ver. Inv. Vet. Perú, 23 (2): 160-170, 2012. DOI: http://dx.doi.org/10.15381/rivep.v23i2.896.

SILVA, F. A. S. ASSISTAT: Versão 7.7 beta. DEAG-CTRN-UFCG - Atualizado em 03 de abril de 2016. Disponível em: <http://www.assistat.com>. Acessado em: 10 de fevereiro de 2017.

TEKELI, A.; ÇELIK, L.; KUTLU, H. R.; GORGULU, M. Effect of dietary supplemental plant extracts on performance, carcass characteristics, digestive system development, intestinal microflora and some blood parameters of broiler chicks. In: XII EPC, Verona, Itália, 2006.

TRAESELI, C. K.; LOPES, S. T. A.; WOLKMER, P.; SCHMIDT, C.; SANTURIO, J. M.; ALVES, S. H. Óleos essenciais como substituintes de antibióticos promotores de crescimento em frangos de corte: perfil de soroproteínas e peroxidação lipídica. Ciência Rural, Santa Maria, v.41, n.2, p.278-284, fev, 2011. https://doi.org/10.1590/S0103-84782011000200016.

YAMAGUCHI, N.; SATOH-YAMAGUCHI, K.; ONO, M. In vitro: evaluation of antibacterial, anticollagenase, and antioxidant activities of hop components Humulus lupulus addressing acne vulgaris. Phytomedicine, v.16, p.369-376, 2009. https://doi.org/10.1016/i.phymed.2008.12.021.

ZHANG, G. F.; YANG, Z. B.; WANG, Y.; YANG, W. R.; JIANG, S. Z.; GAI, G. S. Effects of ginger root (Zingiber officinale) processed to different particle sizes on growth performance, antioxidant status, and serum metabolites of broiler chickens. Poultry Science, 88:2159-2166, 2009. https://doi.org/10.3382/ps.2009-00165. 